\title{
Effect of organ donation after circulatory determination of death on number of organ transplants from donors with neurologic determination of death
}

\author{
Vivek Rao MD PhD, Sonny Dhanani MD, Janet MacLean RD MBA, Clare Payne RN, Elizabeth Paltser MSc, \\ Atul Humar MD, Jeffrey Zaltzman MD
}

n Cite as: CMAJ 2017 September 25;189:E1206-11. doi: 10.1503/cmaj.161043

See related article at www.cmaj.ca/lookup/doi/10.1503/cmaj.170988

\begin{abstract}
BACKGROUND: To increase the available pool of organ donors, Ontario introduced donation after circulatory determination of death (DCD) in 2006. Other jurisdictions have reported a decrease in donations involving neurologic determination of death (NDD) after implementation of DCD, with a drop in organ yield and quality. In this study, we examined the effect of DCD on overall transplant activity in Ontario.
\end{abstract}

METHODS: We examined deceased donor and organ transplant activity during 3 distinct 4-year eras: pre-DCD (2002/03 to $2005 / 06)$, early DCD (2006/07 to $2009 / 10)$ and recent DCD (2010/11 to
2013/14). We compared these donor groups by categorical characteristics.

RESULTS: Donation increased by 57\%, from 578 donors in the pre-DCD era to 905 donors in the recent DCD era, with a $21 \%$ proportion (190/905) of DCD donors in the recent DCD era. However, overall NDD donation also increased. The mean length of hospital stay before declaration for NDD was 2.7 days versus 6.0 days before withdrawal of life support and subsequent asystole in cases of $D C D$. The average organ yield was 3.73 with NDD donation versus 2.58 with $\mathrm{DCD}(p<0.001)$. Apart from hearts, all organs from DCD donors were suc- cessfully transplanted. From the pre$D C D$ era to the recent $D C D$ era, transplant activity in each era increased for all solid-organ recipients, including heart (from 158 to 216), kidney (from 821 to 1321), liver (from 477 to 657 ) and lung (from 160 to 305 ).

INTERPRETATION: Implementation of DCD in Ontario led to increased transplant activity for all solid-organ recipients. There was no evidence that the use of DCD was pre-empting potential NDD donation. In contrast to groups receiving other organs, heart transplant candidates have not yet benefited from DCD.
$\mathbf{F}$

or all candidates waiting for a solid-organ transplant, the lack of suitable deceased donors has led to waiting-list deaths, with mortality rates dependent upon organ type. As a result, donation criteria have been expanded in an effort to increase the deceased-donor pool for all organs. ${ }^{1}$ Initially, efforts focused on accepting organs from increasingly older donors., ${ }^{1,2}$ Surgical techniques have also evolved, allowing a single donation to provide life-saving transplants to multiple recipients, through so-called split-organ transplants. ${ }^{3}$ More recently, the development of ex vivo organ perfusion systems has permitted evaluation, resuscitation and ultimately successful use of marginal organs previously considered unsuitable for transplant. ${ }^{4}$

The most important development in efforts to expand the donor pool has involved donation after circulatory determination of death (DCD)..$^{5}$ In these cases, life-sustaining therapy is withdrawn and organs are recovered after death is declared by absence of circulation. In contrast, traditional organ recovery follows neurologic determination of death (NDD) by standardized criteria, in the setting of intact circulation. DCD has gained prominence with availability of improved techniques for preservation of organs with prolonged ischemia after circulation stops. Initially reported for kidney recipients, successful transplants from DCD donors have now been performed with lung, liver and, most recently, heart. ${ }^{6-10}$

In the setting of this advance in organ donation, concerns have been raised about the quality and yield of organs from $D C D$ and about whether an active DCD program replaces organs that would otherwise have been procured through NDD donation. ${ }^{11,12}$ 
Since 2002, Ontario (Canada's most populous province) has been served by a single organ procurement agency, the Trillium Gift of Life Network (www.giftoflife.on.ca). This network launched a DCD program in 2006, with uptake in most large tertiary centres by 2010. The Trillium Gift of Life Network prospectively tracks all donor and transplant activity in the province, which allows assessment of the introduction of the DCD program on overall organ yield and transplant numbers.

In this study, we compared transplant activity in 3 distinct eras (pre-DCD, early DCD and more recently, when DCD activity has matured) in an attempt to answer the following fundamental questions: Did the introduction of DCD adversely affect potential NDD donation and the overall number of organ donors and transplants? Does DCD yield the same number of organs as traditional NDD donation?

\section{Methods}

All patients listed for solid-organ transplant in the province of Ontario are registered with the Trillium Gift of Life Network. The network is also responsible for accepting referrals for potential organ donors, and it tracks and records all organ procurement in the province. We compared donor and transplant data from 3 distinct 4-year eras: pre-DCD (2002/03 to 2005/06), early DCD (2006/07 to 2009/10) and recent DCD (2010/11 to 2013/14) (with fiscal years beginning in April and ending in March).

To determine whether DCD activity influenced NDD activity, we examined the cause of death for each donor group and the time from hospital admission to declaration of death for each organ donor. We hypothesized that if DCD was pre-empting potential NDD donation, the time from admission to declaration of death would be shorter in the DCD group. We also studied the number of organs transplanted from NDD and DCD donors.

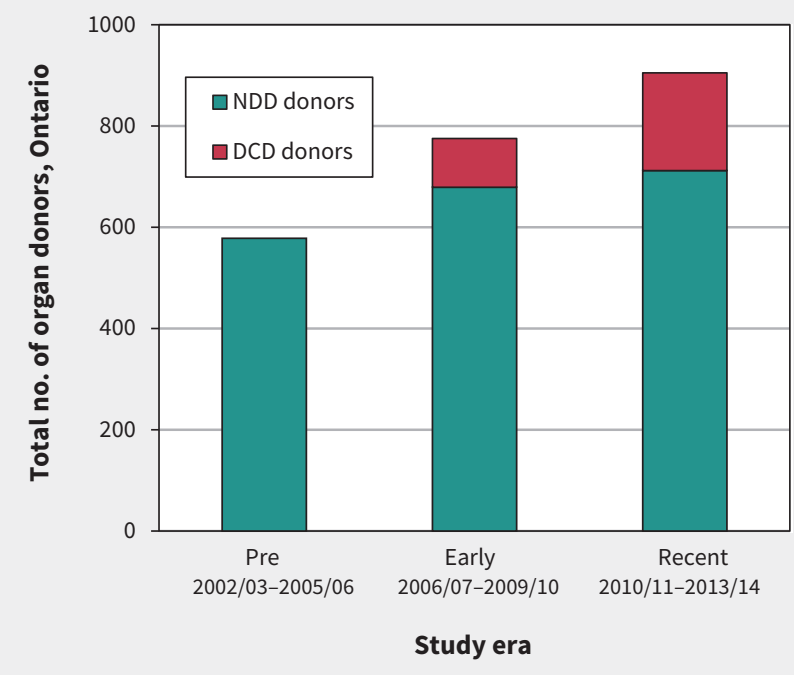

Figure 1: Total number of organ donors in Ontario over the 3 eras in this study, categorized by method of determination of death. $D C D=$ donation after circulatory determination of death, $\mathrm{NDD}=$ neurologic determination of death.

\section{Statistical analysis}

Categorical data were compared by the $\chi^{2}$ or Fisher exact test, as appropriate. To examine the simultaneous effect of year and donation group, we employed a 2-sample $t$ test.

\section{Ethics approval}

This study involved de-identified data collected by a provincial agency, and every transplant hospital in Ontario is mandated to provide these data to the Trillium Gift of Life Network. As such, approval of a research ethics board was not required to either receive the data or publish them. The study was approved by the medical director of research of the Trillium Gift of Life Network (S.D.).

\section{Results}

\section{Overall organ donation}

During the overall study period (April 2002 to March 2014), the total number of organ donors increased from 132 to 223 per year, with a cumulative total of 2258 deceased donors and 6763 deceased-donor transplants. There were more organ donors for each successive era: 578 in the pre-DCD era, 775 in the early DCD era and 905 in the recent DCD era. Figure 1 shows this growth in organ donation, as well as the increasing proportion of DCD donors over the 3 successive eras, with DCD accounting for $21 \%$ (190/905) of overall donor activity in the most recent era, ending in March 2014. Although the proportion of DCD donors increased from 2002 to 2014, a statistical analysis limited to eras 2 and 3 showed no significant change by year, because most organs still originated from NDD donors $(p=0.1)$.

On average, the yield from each NDD donor was 3.73 organs, whereas the yield from each DCD donor was 2.58 organs $(p<0.001)$ (Table 1). The average organ yield from a DCD donor declined from 2.67 in the early DCD era to 2.30 in the recent DCD era $(p=0.3)$.

\section{Donor characteristics}

Overall, NDD donors were older than DCD donors (47 v. 41 years, $p<0.001$ ). Table 2 shows the donor cause of death in the NDD and DCD groups. For NDD donors, cerebrovascular accident or stroke was the most common cause of death, and there was a striking increase in the proportion of deaths from anoxia in the most recent era. In contrast, for DCD donors, anoxia has emerged as the most common cause of death. Over time, the overall number of donors with anoxic brain death has increased $(p<0.001)$.

Figure 2 shows the times from donor admission to hospital to the declaration of death. For most NDD donors (1268/1709 [74\%]), death was declared within 3 days of hospital admission (mean $2.7 \mathrm{~d}$, standard deviation [SD] $3.3 \mathrm{~d}$, range 0-48 d). In contrast, DCD donors were admitted for an average of 6.0 (SD 5.2) days before withdrawal of life support and subsequent asystole (range $0-49 \mathrm{~d}, p<0.001$ ).

\section{Organ transplants}

Table 1 shows the donor rate per 100000 population over the study period, and Figure 3 provides organ-specific transplant rates for NDD and DCD donors. The overall increase in the number of transplants from 2006 to 2014 is almost entirely due to the growth in DCD transplants. There was also an increase in the number of transplants 
from NDD donors over time. There was modest growth in all solidorgan transplants from NDD donors, with a large increase in the number of kidney transplants from DCD donors. Figure $3 C$ shows organ-specific transplants from all donors and confirms that the growth in kidney transplants from DCD donors did not come at the expense of other organs. More specifically, from the pre-DCD era to the recent DCD era, transplant activity in each era increased for all solid-organ recipients, including heart (from 158 to 216), kidney (from 821 to 1321), liver (from 477 to 657) and lung (from 160 to 305).

\section{Interpretation}

Donation after circulatory determination of death was introduced in Ontario in 2006. Certain organ transplant groups, particularly those involved in renal transplant, saw DCD as a potential means to substantially increase the donor pool; others, such as the cardiac transplant groups, feared that the expansion of DCD would come at the expense of traditional NDD transplants. Combined with the previously documented lower organ yield from DCD, there was a legitimate concern that overall transplant numbers would be adversely affected. ${ }^{11,12}$ In the current study, we have shown that NDD donation did not decline in Ontario, despite a substantial increase in DCD over 3 eras. Ontario now enjoys one of the highest rates of DCD in North America. ${ }^{12}$

In jurisdictions such as the United States, where different organ procurement agencies may have overlapping geographic territories, it may be difficult to assess accurately the effects of introducing new donation practices. In contrast, the Trillium Gift of Life Network in Ontario (which has a population of 13 million)

Table 1: Donor characteristics

Donor group; no. of donors $†$

\begin{tabular}{|c|c|c|c|c|}
\hline Characteristic $^{\star}$ & $\begin{array}{c}\text { NDD } \\
n=1969\end{array}$ & $\begin{array}{c}\text { DCD } \\
n=289\end{array}$ & $\begin{array}{c}\text { All } \\
n=\mathbf{2 2 5 8}\end{array}$ & $p$ value \\
\hline Donor rate, per 100000 & & & & $<0.001$ \\
\hline Pre-DCD & 1.18 & NA & 1.18 & \\
\hline Early DCD & 1.33 & 0.19 & 1.52 & \\
\hline Recent DCD & 1.34 & 0.36 & 1.70 & \\
\hline \multicolumn{5}{|c|}{ Organ yield, mean per donor } \\
\hline Overall & 3.73 & 2.58 & 3.58 & $<0.001$ \\
\hline Pre-DCD & 3.51 & NA & 3.51 & \\
\hline Early DCD & 3.75 & 2.67 & 3.61 & \\
\hline Recent DCD & 3.53 & 2.30 & 3.26 & \\
\hline Age, yr, mean & & & & $<0.001$ \\
\hline Pre-DCD & 44.8 & NA & 44.8 & \\
\hline Early DCD & 45.2 & 41.8 & 44.8 & \\
\hline Recent DCD & 47.0 & 41.4 & 45.8 & \\
\hline Cause of death & & & & $<0.001$ \\
\hline Stroke & 1035 & 72 & 1107 & \\
\hline Head trauma & 482 & 84 & 566 & \\
\hline Anoxia & 308 & 118 & 426 & \\
\hline Other & 144 & 15 & 159 & \\
\hline Sex & & & & 0.001 \\
\hline Male & 1108 & 192 & 1300 & \\
\hline Female & 861 & 97 & 958 & \\
\hline Blood group & & & & 0.8 \\
\hline A & 753 & 114 & 867 & \\
\hline B & 222 & 32 & 254 & \\
\hline 0 & 919 & 135 & 1054 & \\
\hline$A B$ & 75 & 8 & 83 & \\
\hline
\end{tabular}

Note: $\mathrm{DCD}=$ donation after circulatory determination of death, $\mathrm{NA}=$ not applicable, NDD $=$ neurologic determination of death.

*The 4-year eras of the study are defined as follows: pre-DCD $=2002 / 03$ to $2005 / 06$, early DCD $=2006 / 07$ to $2009 / 10$, recent DCD $=2010 / 11$ to $2013 / 14$, with all fiscal years beginning in April and ending in March.

tExcept where indicated otherwise. 
oversees all donation and transplant activity within the province. Therefore, this network has a greater ability to assess the effect of a new donor procurement strategy.

An analysis of the donor characteristics shown in Table 2 and Table 3 suggests that DCD donors are distinct from traditional NDD donors. NDD donors were more likely to have experienced an embolic or hemorrhagic neurologic event, whereas DCD donors were more likely to have experienced anoxic brain injury.
Of importance, the mean length of stay before declaration of death was 2.7 days for NDD donors versus 6.0 days to withdrawal of life support and asystole in DCD donors. Thus, death was being declared for potential DCD donors well after the time window for potential NDD donation, with little overlap. This result contradicts a widely held notion that organs are recovered from DCD donors before they become eligible for traditional NDD donation.

\section{Table 2: Cause of death in each donor group}

Cause of death; no. of donors $\dagger$

\begin{tabular}{|c|c|c|c|c|c|}
\hline Era* & CVA or stroke & Head trauma & Anoxia & Other $¥$ & Total \\
\hline \multicolumn{6}{|l|}{ NDD donors } \\
\hline Pre-DCD & 309 & 174 & 45 & 50 & 578 \\
\hline Early DCD & 366 & 168 & 85 & 60 & 679 \\
\hline Recent DCD & 360 & 140 & 178 & 34 & 712 \\
\hline Total, no. (\%) of NDD donors & $1035(53)$ & $482(24)$ & $308(16)$ & $144(7)$ & 1969 \\
\hline \multicolumn{6}{|l|}{ DCD donors } \\
\hline Pre-DCD & NA & NA & NA & NA & NA \\
\hline Early DCD & 30 & 28 & 35 & 3 & 96 \\
\hline Recent DCD & 42 & 56 & 83 & 12 & 193 \\
\hline Total, no. (\%) of DCD donors & $72(25)$ & $84(29)$ & $118(41)$ & 15 (5) & 289 \\
\hline
\end{tabular}

A: NDD

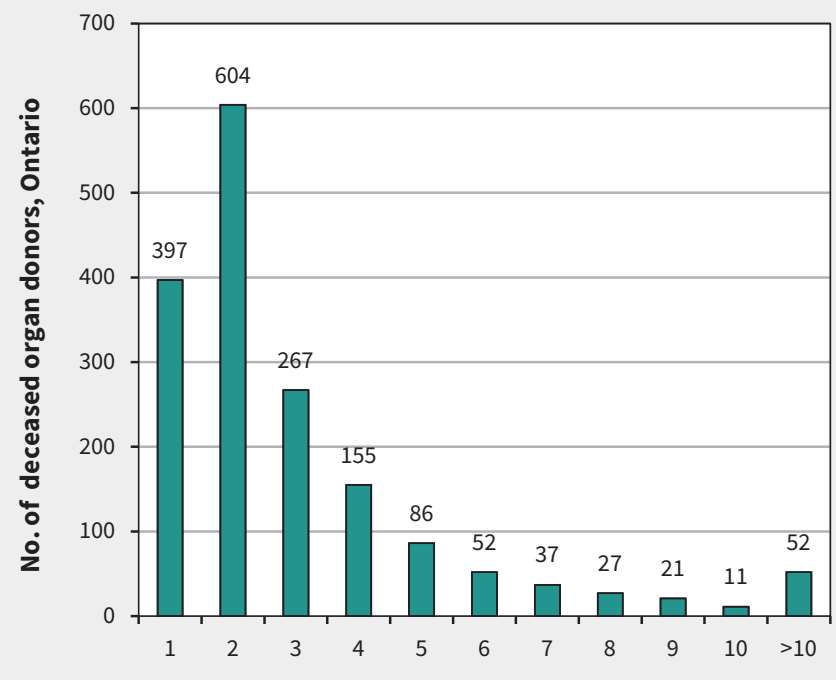

B: DCD

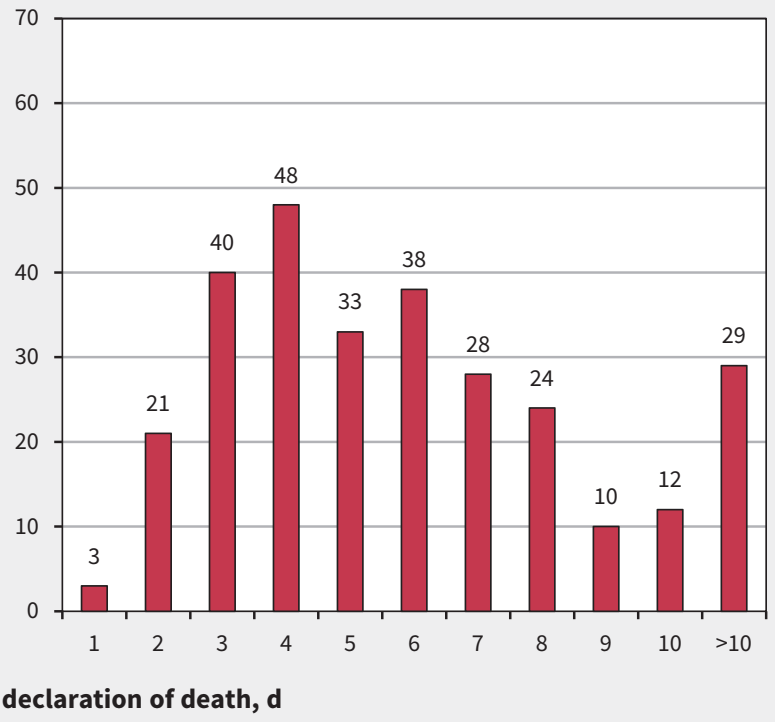

Figure 2: Time from hospital admission to neurologic determination of death (NDD; panel A) and withdrawal of life support for donation after circulatory determination of death (DCD; panel B). 
Our data clearly support previous reports that overall yield from DCD donors is less than that from NDD donors. At present, this is likely because hearts are not currently procured from $D C D$ donors; however, the introduction of ex vivo perfusion strategies and the successful use of DCD hearts in other jurisdictions may result in equalization of the organ yields between donor groups..$^{10}$ The largest overall growth in transplants was for kidneys. Similarly, kidneys had the most substantial increase in DCD transplants. In contrast, growth in heart transplants has been modest, but, importantly, the number of heart transplants did grow over time, which suggests that DCD has not negatively affected transplants of this organ. Important advances in heart transplant after DCD have occurred elsewhere, ${ }^{10}$ and this work should be replicated in North America. Perhaps more relevant to the cardiac transplant groups is the continued development of mechanical assist devices, which are increasingly being offered as an alternative to transplant. ${ }^{13}$ The introduction of ex vivo perfusion technologies has affected the number of DCD lung transplants, and we suspect that the same will occur with heart transplants once ex vivo cardiac perfusion becomes widely available. ${ }^{9,14}$

An unanswered question from this study relates to the quality of the organs from DCD and NDD donors. Wadei and associates ${ }^{11,14}$ and Orman and colleagues ${ }^{12}$ reported that use of both renal and liver grafts from DCD donors led to inferior outcomes relative to NDD donors. We have previously reported on transplant outcomes after the first 3 years of the Ontario DCD experience, ${ }^{15}$ and found that recipients of organs from DCD donors had survival similar to that of recipients of organs from NDD donors. The current study did not examine post-transplant outcomes, but was primarily designed to look at donation activity and the potential effects of DCD on NDD activity. Although organ quality is an important consideration, it must be evaluated in the context of expansion of the overall donor pool, much as has occurred with the use of "extended criteria" organs from donors with advanced age or other relative contraindications to donation. ${ }^{1,2}$

\section{Limitations}

It is difficult to speculate on what the growth rate of NDD donation would have been in the absence of DCD. We concede that this study is observational in nature and that we cannot ascribe causality to the changes observed. Nonetheless, we believe that these results show that overall NDD activity did not suffer from the introduction of a

\section{A: NDD donors}

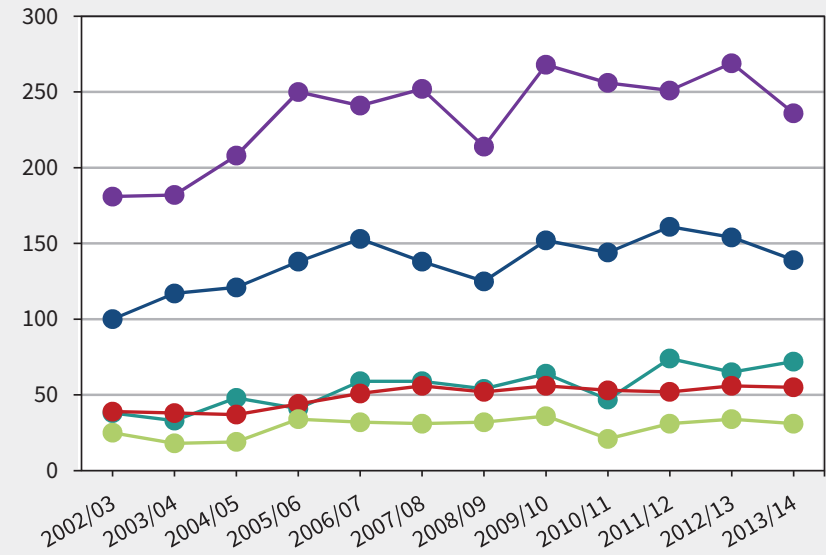

B: DCD donors

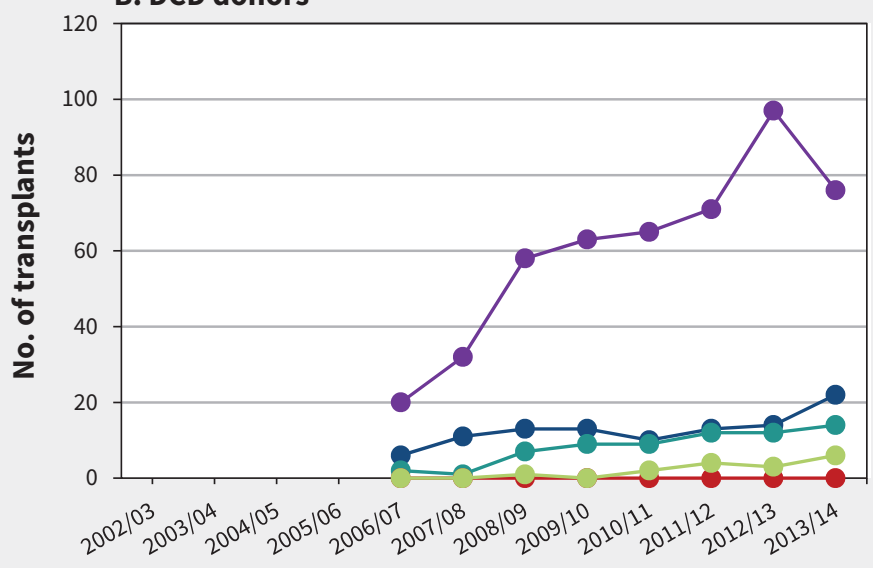

C: All donors

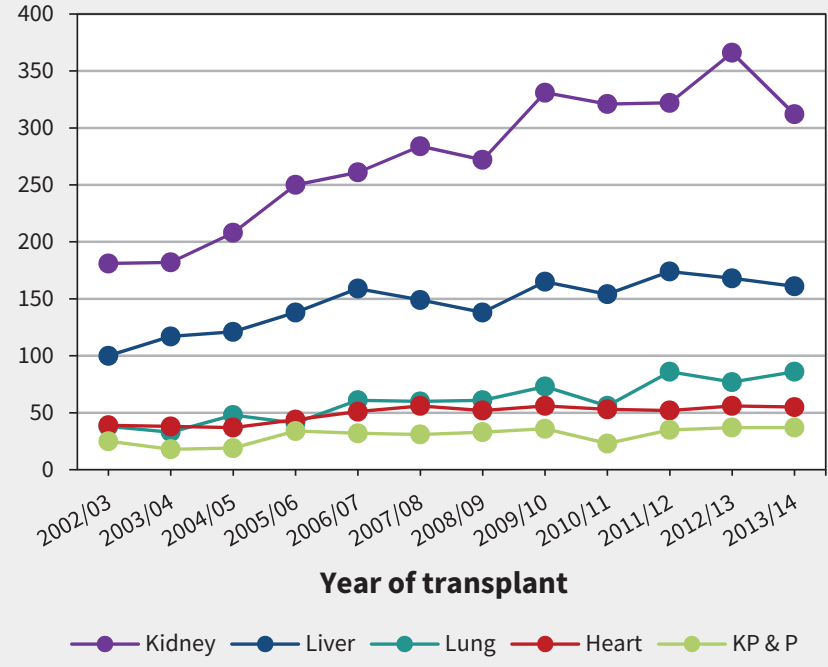

Figure 3: Organ-specific transplants from donors after neurologic determination of death (NDD; panel A) and from donors after cardiac determination of death (DCD; panel B). Panel $C$ presents organ-specific transplants from all donors. The vertical scale differs across the three panels. KP\&P = kidney-pancreas and pancreas. 
DCD program (in contrast to other reports in the literature ${ }^{7,8,12}$ ). It is unlikely (given historical rates) that growth in NDD donation would have matched or exceeded that of DCD. Our data also suggest that DCD donors are unlikely to transition to conventional NDD donors; thus, the introduction of DCD has had the intended effect of expanding the donor pool and increasing overall transplant activity, without adversely affecting NDD donation.

Other initiatives may have simultaneously increased NDD donation, thus negating any potential identifiable loss caused by DCD practice. These initiatives include the introduction of donor physicians, improved donor referral policies and the introduction of extended donor criteria. Our study could not take into account changes in health policy or access to care that may have influenced donor and transplant activity during the period of our study. However, it is likely that any initiative, including promotion of DCD, would benefit both DCD and NDD donation equally.

Our study reflects transplant activity in a single jurisdiction, and the results may not be generalizable to other regions. Relative rates of donation and transplant in different jurisdictions are multifactorial and can be difficult to compare. Our focus on Ontario was necessary to ensure homogeneity of the data. However, other regions in Canada and the US should be able to use our findings to reflect on their own inherent assumptions toward DCD.

\section{Conclusion}

Donation after circulatory determination of death has had a positive effect in Ontario in terms of both overall number of donors and transplant activity. Donation after NDD does not appear to have been adversely affected. Although there are disparities among organ groups, we foresee that an active DCD program will continue to have a positive effect for all solid-organ transplant recipients.

Future work is needed to investigate why NDD rates continue to increase and also to investigate reasons for the success of DCD. Further investigation is also needed to identify why death is declared earlier for NDD donors than for DCD donors and to determine the important factors involved with neuroprognostication before DCD is considered.

\section{References}

1. Bernhardt AM, Reichenspurner H. High-risk donors: extending our criteria in times of organ shortage. Curr Opin Organ Transplant 2014;19:494-9.

2. Prieto $D$, Correia $P$, Baptista $M$, et al. Outcome after heart transplantation from older donor age: expanding the donor pool. Eur J Cardiothorac Surg 2015;47:672-8.

3. Renz JF, Emond JC, Yersiz H, et al. Split-liver transplantation in the United States: outcomes of a national survey. Ann Surg 2004;239:172-81.

4. Lim GB. Transplantation: ex-vivo perfusion of human hearts - implications for donor organ availability. Nat Rev Cardiol 2015;12:317.

5. Weber M, Dindo D, Demartines N, et al. Kidney transplantation from donors without a heartbeat. N Engl J Med 2002;347:248-55.

6. Machuca TN, Mercier O, Collaud S, et al. Lung transplantation with donation after circulatory determination of death donors and the impact of ex-vivo lung perfusion. Am J Transplant 2015;15:993-1002.

7. Johnson RJ, Bradbury LL, Martin K, et al.; UK Transplant Registry. Organ donation and transplantation in the UK - the last decade: a report from the UK national transplant registry. Transplantation 2014;97 Suppl 1:S1-27.

8. Bendorf A, Kelly PJ, Kerridge IH, et al. An international comparison of the effect of policy shifts to organ donation following cardiocirculatory death (DCD) on donation rates after brain death (DBD) and transplantation rates. PLoS One 2013;8:e62010.

9. Watson CJE, Dark JH. Organ transplantation: historical perspective and current practice. Br J Anaesth 2012;108:i29-42.

10. Dhital KK, lyer A, Connellan M, et al. Adult heart transplantation with distant procurement and ex-vivo preservation of donor hearts after circulatory death: a case series. Lancet 2015;385:2585-91.

11. Wadei HM, Heckman MG, Rawal B, et al. Comparison of kidney function between donation after cardiac death and donation after brain death kidney transplantation. Transplantation 2013;96:274-81.

12. Orman ES, Barritt AS IV, Wheeler SB, et al. Declining liver utilization for transplantation in the United States and the impact of donation after cardiac death. Liver Transpl 2013;19:59-68.

13. Sutcliffe P, Connock M, Pulikottil-Jacob R, et al. Clinical effectiveness and costeffectiveness of second and third generation left ventricular assist devices as either bridge to transplant or alternative to transplant for adults eligible for heart transplant: systematic review and cost-effectiveness model. Health Technol Assess 2013;17:1-499.

14. Wadei HM, Bulatao IG, Gonwa TA, et al. Inferior long-term outcomes of liverkidney transplantation using donation after cardiac death donors: singlecenter and organ procurement and transplantation network analyses. Liver Transpl 2014;20:728-35.

15. Hernandez-Alejandro R, Wall W, Jevnikar A, et al. Organ donation after cardiac death: donor and recipient outcomes after the first three years of the Ontario experience. Can J Anaesth 2011;58:599-605.
Competing interests: Vivek Rao is a member of the board of directors for the Trillium Gift of Life Network, has served on advisory boards for Medtronic and CorMatrix Cardiovascular and is a member of the speakers' bureau for St. Jude Medical. Sonny Dhanani was formerly the chief medical officer for the Trillium Gift of Life Network (2010-2016). No other competing interests were declared.

This article has been peer reviewed.

Affiliations: Division of Cardiovascular Surgery (Rao), Peter Munk Cardiac Centre, University of Toronto, Toronto, Ont.; Division of Critical Care
(Dhanani), Children's Hospital of Eastern Ontario, University of Ottawa, Ottawa, Ont.; Trillium Gift of Life Network (MacLean, Payne, Paltser); Multiorgan Transplant Unit (Rao, Humar), Toronto General Hospital, University of Toronto; Division of Nephrology (Zaltman), St. Michael's Hospital, University of Toronto, Toronto, Ont.

Contributors: Vivek Rao, Sonny Dhanani, Atul Humar and Jeffrey Zaltzman made substantial contributions to the conception and design of the study, and to the acquisition, analysis and interpretation of the data. Janet MacLean, Clare Payne and Elizabeth Paltser retrieved data from the Trillium Gift of Life Network database and performed initial data review and statistical analysis; Elizabeth Paltser performed additional statistical analyses following the initial editorial review. Vivek Rao, Sonny Dhanani, Atul Humar and Jeffrey Zaltzman drafted the manuscript and made revisions before initial submission. All of the authors revised the manuscript for important intellectual content, approved the final version and agreed to act as guarantors of the work.

Accepted: Apr. 20, 2017

Correspondence to: Vivek Rao, vivek.rao@ uhn.ca 